\title{
La production d'articulations et de mouvements pour la santé des hommes au Brésil :
}

La sexualité comme porte d'entrée

Fabíola Rohden

l'Université Fédérale du Rio Grande do Sul (UFRGS)

\section{Resumo}

Esta investigação trata da medicalização da sexualidade masculina no Brasil, centrada na criação de uma nova farmacologia do sexo na passagem para o século XXI, via o foco na disfunção erétil e na chamada andropausa. Destaca-se a nova ênfase na noção de saúde sexual baseada no aprimoramento individual e uso de medicamentos, além da promoção do interesse masculino no desempenho sexual, como porta de entrada para se chegar ao tratamento da saúde do homem. A perspectiva teórica baseia-se na análise antropológica das redes sociotécnicas que circunscrevem estes processos. No que se refere à metodologia, foi realizada pesquisa documental em arquivos históricos, material de imprensa e fontes do governo, além de entrevistas e etnografia em congressos médicos e eventos públicos.

Palavras-chave: medicalização, sexualidade masculina, saúde sexual, disfunção erétil.

\section{Abstract}

This investigation deals with the medicalization of male sexuality in Brazil, focusing on the creation of a new pharmacology of sex in the transition to the Twenty-First Century, directed to treatment of erectile dysfunction and andropause. The article points to an emphasis on the notion of sexual health based on individual improvement and medication, as well as the promotion of interest in male sexual performance, as the gateway to the treatment of 
men's health. The theoretical approach is based on anthropology and social studies of science. With respect to methodology, documentary research was conducted in historical archives and contemporary sources (such as material and press releases from the government), interviews and ethnography in medical conferences and public events.

Keywords: medicalization, male sexuality, sexual health, erectile dysfunction.

\section{Résumé}

Cette recherche s'intéresse à la médicalisation de la sexualité masculine au Brésil en se concentrant sur la création d'une nouvelle pharmacologie du sexe, au tournant du XXI siècle, destinée à traiter la dysfonction érectile et la dite andropause. Elle souligne le nouvel accent mis sur la notion de santé sexuelle fondée sur l'accomplissement personnel et l'utilisation de médicaments et sur la promotion de l'intérêt masculin pour la performance sexuelle comme porte d'entrée vers le traitement de la santé de l'homme. La perspective théorique se base sur l'analyse anthropologique des réseaux sociotechniques sous-tendant ces processus. Outre des entretiens et une étude ethnographique de congrès médicaux et d'événements publics, la méthodologie repose sur une recherche documentaire dans les archives historiques, les matériaux divulgués et les sources gouvernementales.

Mots-clés: Médicalisation, sexualité masculine, santé sexuelle, dysfonction érectile. 


\section{La production d'articulations et de mouvements pour la santé des hommes au Brésil :}

La sexualité comme porte d'entrée

Fabíola Rohden

\section{Les hommes, la sexualité et le bien collectif}

Ces dernières décennies, le Brésil a assisté à un intense processus de médicalisation et pharmacologisation de la sexualité centré sur la création de nouvelles ressources technologiques, de catégories de diagnostics et de formes d'intervention incluant la promotion de politiques publiques. Ce phénomène se déroule selon un scénario où se distinguent des éléments tels que les médicaments, les sociétés médicales, l'industrie pharmaceutique, les médias, les consommateurs et des événements allant de la prescription de médicaments dans un cabinet à la promotion de campagnes et à la mise en place de programmes dans le système public de santé. Il peut être illustré par le biais d'un ensemble d'initiatives centrées sur la promotion du slogan « La santé sexuelle comme portail de la santé de l'homme ». L'idée fondamentale est que les hommes, qui auraient pour tradition de négliger leur santé, pourraient être attirés dans ce système par l'attention sur la fonction érectile.

Ce travail est le résultat d'une recherche visant à cartographier la création et la promotion de diagnostics relatifs au processus de vieillissement des hommes et des femmes dans leur interrelation avec les manifestations associées à la sexualité, en prenant pour référence la dimension des relations de genre. ${ }^{1}$ En ce sens, il a enquêté sur la manière dont ces nouveaux diagnostics

\footnotetext{
$1 \quad$ Projet de recherche Différences de genre dans la récente médicalisation du vieillissement et sexualité : la création des catégories ménopause, andropause et dysfonction sexuelle (sponsorisé par le Conseil National de Recherche - CNPq). Ce travail a été présenté lors du Colloque international Produire du savoir, gouverner des populations - Anthropologie, science studies et politiques de santé. École normale supérieure, Lyon, 2013.

Certaines données analysées ici ont été discutées de façon préliminaire dans Rohden, F. 2012. «Capturados pelo sexo: a medicalização da sexualdidade masculina em dois momentos ". Ciência e Saúde Coletiva, 17: 2645 - 2654.
} 
sont apparus dans le champ médical brésilien ces dernières décennies. Cette recherche à l'empreinte socio-anthropologique a privilégié l'articulation entre différentes techniques de recherche qualitative telle que l'observation participante, les entrevues et la recherche documentaire. Elle a eu recours à l'analyse d'articles de revues scientifiques, de sites de sociétés médicales, de reportages, d'émissions de télévision et des matériaux divulgués dans la presse, outre l'ethnographie de congrès médicaux et de campagnes, et la réalisation d'entretiens avec des professionnels des domaines concernés. La dispersion des sources a été essentielle afin de montrer l'étendue des processus étudiés et les réseaux sociotechniques complexes y participant.

Il convient de souligner que les études visant à cartographier historiquement un intérêt différencié de la médecine, dans sa réflexion théorique et sa pratique quotidienne concernant les hommes et les femmes, sont très récentes. Un panorama préliminaire montre que les femmes ont été bien plus visées par le savoir médical que les hommes même si, ces dernières décennies, ceux-ci jouissent d'une attention spécifique en vertu de la création de nouveaux diagnostics et pathologies (Marshall et Katz 2002; Rosenfeld et Faircloth 2006; Loe 2001; Rohden, 2009).

Le résultat de cette analyse devient plus évident lorsque nous contrastons ce récent processus de médicalisation de la sexualité masculine et le grand mouvement d'intervention du début du XXe siècle, au Brésil, pour affronter la syphilis et lutter, de manière plus générale, contre les maladies vénériennes. Le choix de ces deux processus ne cherche aucunement à affirmer qu'ils sont analogues, car la distance historique et contextuelle impose de nombreuses différences. Cependant, cette opération analytique fait ressortir l'urgence de certaines structures discursives pouvant être comparées de façon productive. Je propose donc que la discussion de ces vecteurs analytiques permet de réfléchir avec une certaine profondeur sur les processus de médicalisation des hommes articulant sexe et santé par le biais d'agencements assez précis en ce qui concerne certaines conceptions normatives implicites.

Au Brésil, le mouvement médico-étatique contre la syphilis des premières décennies du $\mathrm{XX}^{\mathrm{e}}$ siècle était lié à la promotion d'une morale sexuelle scientifique ancrée sur l'autocontrôle masculin. Le corps et la sexualité des hommes ont fait l'objet de grandes inquiétudes en raison des maladies vénériennes. Dans son analyse de la lutte contre la syphilis dans ce pays, Sergio Carrara 
(1996) décrit la gigantesque mobilisation contre cette maladie qui, à partir de l'association avec la dégénération et l'affaiblissement de la race, deviendrait, selon les médecins de l'époque, une menace pour la constitution d'une population saine et pour l'ordre social.

Les investissements concernant la syphilis doivent être compris dans le cadre d'un processus de régulation de la sexualité et d'appréhension envers le futur de la population face aux aspirations de l'État. La construction sociale de la syphilis a alors été articulée autour du processus de configuration sociale de la nation car l'idée centrale en était la dégénération de la race. La lutte contre la syphilis met aussi en évidence la construction d'un nouveau modèle d'individu, capable d'autocontrôle, qualité qui serait nécessaire aux nouvelles structures politiques en cours de constitution. Il est notable que cet autocontrôle individuel se référait principalement aux hommes et au comportement sexuel masculin. Au travers de mesures concernant la syphilis, les médecins et l'État semblaient chercher à atteindre le pouvoir oligarchique et patriarcal, traditionnel au Brésil à l'époque, par le biais de la remise en cause des prérogatives traditionnelles masculines se rapportant à la possibilité de contrôler l'accès aux femmes et, ainsi, aux plaisirs sexuels et aux alliances matrimoniales. Il semblerait que le corps des hommes, jusqu'alors plus impénétrable que celui des femmes, des enfants et des pervers sexuels, ait fini par se rendre à la médicalisation au travers de la perception des dangers de la syphilis (Carrara 1996).

Dans le contexte des débats autour de cette maladie, la fonction reproductive et ses organes étaient vus comme une propriété moins individuelle que collective. En fin de compte, la responsabilité biologique face à la descendance devrait passer au premier plan. Les hommes ont donc été visés à partir d'une maladie qui compromettait leur descendance, même si, plus immédiatement, elle avait une incidence sur la dégradation individuelle ellemême. La science des problèmes sexuels masculins qui se développait alors était surtout liée à une maladie externe, venue de l'extérieur, et due à «l'excès sexuel».

Un chapitre intéressant de ce processus de médicalisation de la sexualité, ou en un certain sens, des résistances qu'il a suscitées, est celui des tentatives du médecin José de Albuquerque pour promouvoir l'éducation sexuelle et la création de l'andrologie dans les années 1930. Toujours selon Carrara, ce 
médecin a été responsable d'initiatives telles que la fondation du Cercle brésilien d'éducation sexuelle en 1933 et du Bulletin d'éducation sexuelle, publié bimestriellement entre 1933 et 1939 et distribué gratuitement sur tout le territoire national, qui a atteint un tirage de 100 ooo exemplaires. Entre autres activités, comme exposés et conférences radiophoniques, ce Cercle a organisé la Semaine d'éducation sexuelle, en 1934 et la Journée du sexe, le 20 novembre 1935, soutenant que le sexe devrait être soumis à une " morale scientifique " (Carrara 1996).

Ce médecin, qui s'autoproclamait sexologue, s'est aussi investi dans la création de l'andrologie, une spécialité qui serait consacrée exclusivement aux problèmes de la « fonction sexuelle » et de « l'appareil reproducteur masculin ". Dans cette même ligne, Albuquerque a fondé le Jornal de Andrologia (Journal d'Andrologie) en 1932, édité jusqu'en 1938, également distribué gratuitement, qui a atteint un tirage de 30000 exemplaires en 1935, année où il a commencé à être publié en cinq langues différentes. Son engagement a également fait naître une chaire de clinique andrologique à l'Université du District Fédéral, dont il a été le titulaire de 1936 à 1938, avant de démissionner en raison des problèmes soulevés par la nomination du catholique Alceu Amoroso Lima au poste de recteur de cette université. Dans la dispute entre spécialités médicales, il combattait aussi les urologues qui lui semblaient monopoliser indûment des problèmes tels que l'impuissance, la stérilité, l'éjaculation précoce et les maladies vénériennes, entre autres, lesquels devraient faire l'objet de traitements plus appropriés par l'andrologie (Carrara 1996).

Malgré le fort investissement de l'État et des organisations médicales, cet effort n'a pas suffi à consolider des politiques publiques consacrées spécifiquement à la santé de la population masculine, au contraire de ce qui a eu lieu à partir des grandes initiatives consacrées aux femmes (Rohden 2009).

\section{La production d'un nouveau scénario au XXI ${ }^{\mathrm{e}}$ siècle : sexualité masculine et santé publique}

Le panorama décrit jusqu'ici contraste profondément avec les processus instaurés au tournant du XXIe siècle, qui impliquent de nouveaux investissements médico-étatiques consolidés et associés à la médicalisation de la masculinité. Ce phénomène serait représenté de façon exemplaire par la création 
de la Politique nationale d'attention intégrale à la santé de l'homme (PNAISH) officiellement mise en œuvre par le gouvernement brésilien en août 2009 et marquée par la pression des urologues face au gouvernement et à la société civile, comme le prouvent les diverses campagnes publiques et la divulgation dans les médias. Son accent retombe sur la notion de santé sexuelle et sur la promotion des diagnostics récents de dysfonction sexuelle et d'andropause (Déficit androgénique lié à l'âge), en étroite articulation avec l'engagement des industries pharmaceutiques pour encourager la consommation de médicaments associés à ces « dysfonctions » sur le marché brésilien (Carrara, Russo et Faro 2009).

Pou autant que la PNAISH soit le résultat d'une série d'intérêts, parmi les principaux agents ayant contribué à sa création se retrouve un groupe d'urologues très actif publiquement, représentés par la Société brésilienne d'urologie (SBU). Depuis 2004, celle-ci réalisait des campagnes et exerçait des pressions sur certains secteurs du gouvernement et sur des parlementaires, des conseillers de santé et d'autres sociétés médicales pour élaborer une politique dans cette direction. En 2008, cette initiative s'est concrétisée par la signature d'un accord de coopération technique entre la SBU et le Ministère de la Santé visant à promouvoir l'assistance à l'homme dans le système public de santé, par le biais de l'orientation des médecins et la divulgation de campagnes d'informations, dont la première, entre juillet et septembre 2008, a été consacrée à la dysfonction érectile.

Au-delà de la discussion plus profonde autour de la PNAISH (Carrara, Russo et Faro 2009; Gomes 2011; Medrado, Lyra et Azevedo 2009, 2011) et des processus contemporains de mise en œuvre d'une attention spéciale à la santé de l'homme, cible d'une série d'études (Schreiber, Gomes et Couto 2005; Schraiber et Figueiredo 2011; Gomes, Schraiber et Couto 2011; Pinheiro, Couto e Silva 2011; Couto, Pinheiro et Valança 2010), l'objectif est ici de se concentrer de façon privilégiée sur les associations entre sexualité et médicalisation (Gomes 2008). Pour ce faire, nous partons d'une citation qui est devenue de plus en plus récurrente et qui a marqué les événements récents autour de la médicalisation de la santé masculine, du moins en ce qui concerne les appels faits par les urologues et leurs entités associatives : le slogan « La santé sexuelle en tant que portail de la santé de l'homme ». Il n'est nullement question de chercher l'origine, la création, ni même les intentions primaires 
de cette formule, mais d'observer comment il est de plus en plus utilisé et sert à promouvoir un ton spécifique pour ce qui est de l'articulation entre sexe, santé et médicalisation.

En 2008, cette idée était couramment propagée dans des reportages de presse, des matériaux mis au point par les laboratoires pharmaceutiques, des congrès médicaux et des événements publics. Ce thème apparaît à la une de revues à grand tirage comme Veja et Isto é, dont, par exemple, celle de la Section Spéciale du 19 mars intitulée « La Révolution bleue : dix ans après le lancement du Viagra, l'impuissance sexuelle n'est plus un fantôme masculin ». Parmi les principaux bénéfices du Viagra mentionnés, nous citerons une plus grande facilité de diagnostic de l'hypertension et du diabète, problèmes dont l'un des symptômes peut être la dysfonction érectile. Cet article met l'accent sur la publicisation du thème de la dysfonction érectile et de l'utilisation du Viagra en tant que porte d'entrée vers la santé de l'homme (Buchalla 2008). La revue Isto É du 9 juillet 2008 traite de la création de la PNAISH en mettant en avant l'argument de ce que la dysfonction érectile pourrait entraîner des perturbations dans la vie des hommes et indiquer également la présence d'autres problèmes de santé, comme les maladies cardio-vasculaires. Dans le reportage du journal O Globo du 17 août 2008, le ton est le même, comme le montrent les affirmations de l'urologue José Carlos de Almeida, alors président de la Société Brésilienne d'Urologie (SBU), pour qui la dysfonction érectile est rarement la cause d'un problème, mais fondamentalement la conséquence d'autres maladies.

Dans les congrès médicaux, ce thème était également en évidence, et dans les exposés et dans les matériaux préparés par les laboratoires pharmaceutiques (Faro et al 2013). L’entreprise Bayer Schering Pharma, qui était particulièrement engagée à se promouvoir en tant que " premier laboratoire ayant une gamme axée sur la santé de l'homme ", a distribué, lors du Congrès International d'Urologie qui s'est tenu à Rio de Janeiro en 2008 et lors du $10^{e}$ Congrès de la Société Latino-Américaine de Médecine Sexuelle, réalisé à Florianópolis, un même document intitulé « La santé sexuelle comme portail de la santé de l'homme ». Celui-ci comprenait trois articles : « La santé sexuelle comme portail de la santé de l'homme » de Ricardo Meirelles, (professeur d'endocrinologie à l'université PUC-Rio et président du Département d'endocrinologie féminine e d'andrologie de la Société brésilienne d'endocrinologie métabolique) ; « Déficit de testostérone et dysfonction effective en 
tant que composantes du syndrome métabolique ", de Farid Saad (professeur honoraire à la Hang Tuah University, Indonésie); et « Le rôle de la thérapie de recomposition de testostérone dans la santé sexuelle et somatique masculine ", de Svetlana Kalichencko (Présidente du Département d’andrologie et d'urologie du Scientific Research Center for Innovations et leader de la filiale russe de l'International Society for the Study of Aging Males). Ce document " réservé à la classe médicale ", qui renforçait l'association entre performance sexuelle, jeunesse et santé de l'homme, portait en couverture interne une publicité du Nebido, injection trimestrielle de testostérone indiquée pour le traitement du Déficit androgénique lié à l'âge (DALA).

Dans la Campagne nationale d'information sur la santé de l'homme mise en œuvre par la SBU à partir d'août 2008, l'accent retombait également sur la dysfonction érectile, sous prétexte que celle-ci est un important indicateur de maladies, car elle peut être liée à des cardiopathies, à l'hypertension et au diabète. Le site de la SBU mettait en avant que la « Dysfonction érectile est le thème de l'action, car il s'agit d'un indicateur de maladies. Ce problème atteint près de 50\% des hommes de plus de 40 ans. Moins de $10 \%$ consultent leur médecin. Notre entité veut éviter l'automédication. " C'était également la marque des vidéos que la SBU divulguait à l'époque. C'était aussi le thème de la première émission de la TVSBU qui consistait en une conférence sur la Dysfonction érectile de l'éminent urologue Sidney Glina où, entre autres aspects, il soulignait que derrière la dysfonction érectile se cachaient d'autres maladies. Dans la série d'émission Cidadão Saudável (Citoyen en bonne santé), le lien entre la dysfonction érectile et d'autres maladies était aussi abordé, cette fois-ci par Antônio Barbosa de Oliveira (http://www.sbu.org.br).

Par ailleurs, il est intéressant de noter la façon dont cette notion serait présente lors d'événements liés à la création de la PNAISH. C'est le cas du $4^{\mathrm{e}}$ Forum de politiques publiques et santé de l'homme organisé par la Commission de sécurité sociale et famille, de la Chambre des Députés, réalisé le 7 août 2008, à Brasilia. L'audience publique avait pour thème " La santé de l'homme, aspects urologiques et le SUS - Système Unique de Santé : situation et perspectives ». Parmi les discussions réalisées, Sidney Glina se distingue de nouveau avec sa communication « Dysfonction érectile - abordage et traitement. Le Système unique de santé doit-il distribuer ces médicaments gratuitement ? " Il y évoque la dysfonction érectile comme question de santé publique et comme indicateur d'autres maladies à partir d'une série de 
références bibliographiques, de données épidémiologiques et de documents internationaux pour souligner le fait que le " traitement de la Dysfonction érectile peut être la porte d'entrée de l'homme vers le Système de Santé ! " (www2.camara.gov.br/internet/homeagencia/matérias.html).

Dans les années suivantes, cette même perspective continuerait présente de forme significative dans ce scénario d'action publique des urologues, comme lors du $5^{\mathrm{e}}$ Forum de politiques publiques et santé de l'homme organisé par la Commission de sécurité sociale et famille de la Chambre des Députés, le 20 août 2009. Dans une entrevue sur cet événement pour l'Agência Câmara de Notícias, Sidney Glina disait que l'urologie « est une porte d'entrée vers le SUS, où le patient peut commencer à fréquenter des médecins d'autres spécialités, comme des cardiologues. " (www2.camara.gov.br/internet/ homeagencia/matérias.html) Ce médecin insistait encore sur l’importance, pour le SUS, de disposer de plus d'urologues.

Cette importance surgirait aussi parmi les urologues liés à l'Association brésilienne pour les études des inadéquations sexuelles (ABEIS), organisme important dans la trajectoire de la promotion de la médecine sexuelle au Brésil (Russo et al 2011). L'entrevue que Paulo Brito Cunha, alors président de cette association et membre de la Société brésilienne d'urologie, a concédé à la journaliste Lilian Ribeiro, de la radio CBN, le 15 mai 2010, en est un bon exemple. L'entretien se centre sur la relation entre dysfonction érectile et maladies préexistantes. Dans la première partie, le médecin cherche à mettre en relief la possible association entre médicaments pour la dysfonction érectile et problèmes cardio-vasculaires. Plus encore, il en vient à affirmer que l'utilisation du Viagra serait un bénéfice pour éviter des difficultés de cet ordre.

Il explique ensuite que le premier symptôme apparaissant avant une maladie cardiaque, le diabète ou l'hypertension sont les « troubles de la fonction érectile ". Puis il reprend l'idée de la fonction érectile ainsi que la notion plus vaste de "santé sexuelle », comme " porte d'entrée » vers le diagnostic et le traitement d'autres maladies :

" Vous voulez savoir si vous êtes hypertendus ? Demandez comment va votre situation... fonction érectile. Vous voulez savoir si votre cholestérol est élevé ? Demandez-vous comment va votre fonction érectile. La fonction érectile est comme une porte d'entrée vers cela [...]. Nous avons l'Associação Brasileira para 
o Estudo da Inadequação Sexual, notre site est www.abeis.org.br. Qui s'y rend va trouver la liste des nombreux professionnels travaillant sur ce sujet [...] il liste les personnes les plus compétentes pour le traiter car, comme le dit l'Organisation Mondiale de la Santé, l'une des choses qui qualifie le mieux le niveau de qualité de vie des personnes est leur activité sexuelle. [...] " (www. abeis.org.br).

Soulignons, dans cette citation, la connexion entre le diagnostic d'hypertension et la dysfonction érectile, la mention à l'ABEIS et la promotion de ses associés en tant que professionnels adaptés au diagnostic et au traitement, la référence à l'OMS et la notion d'activité sexuelle, sans parler de celle de " santé sexuelle ", comme meilleur indicateur de qualité de vie. À cela, le président de l'ABEIS ajoute, "sur les bases de la littérature internationale ", que seuls $10 \%$ des hommes ayant des difficultés d'érection demandent une orientation et que les médecins ne sont pas habitués à interroger leurs patients sur leur activité sexuelle. Ces données aident à corroborer l'idée qu'il faut donc attirer l'attention sur ce problème et promouvoir des initiatives concernant aussi bien les possibles patients que les médecins et les secteurs de la santé.

Ainsi, l'une des plus importantes initiatives en relation à la création d'une action publique pour la santé de l'homme a eu lieu de mars à septembre 2010 : le Mouvement pour la santé masculine. Axée sur la population masculine, cette campagne était organisée par la Société brésilienne d’Urologie (SBU) et recevait l'appui financier de l'entreprise pharmaceutique Eli Lilly. Elle comprenait un site web, "Mouvement pour la santé masculine " (www. movimentopelasaudemasculina.com.br), et une tournée appelée "Caravane pour la Santé Masculine ", pour laquelle un semi-remorque a été aménagé en salle de consultation et a visité les 22 villes les plus importantes du Brésil pour fournir gratuitement à la population masculine des orientations médicales sur la Dysfonction érectile (DE), les Maladies de la Prostate et l'Andropause. Le projet incluait encore des spots publicitaires à la télévision et des annonces sur Internet. Son objectif était d'offrir aux hommes des informations et des éclaircissements sur les trois maladies en question, pour sensibiliser cette population aux " problèmes masculins ". Dans les villes visitées, les rencontres se déroulaient dans des lieux publics comme des places, des parcs, des aires de stationnement et des centres commerciaux. Pendant 78 jours, la Caravane pour la Santé Masculine a accueilli plus de 10 mille hommes. Ce chiffre montre l'importance d'une telle initiative et ses 
répercussions possibles sur le plan national, indiquant que cette action peut être considérée comme le vecteur d'un réseau plus vaste.

Le Mouvement pour la santé masculine s'est organisé autour du thème central de l'association entre sexualité et santé. Depuis la mise en place du site web, où la dysfonction érectile occupait une place principale, jusqu'à la manière d'en parler et la description qu'en faisaient les personnes et les membres du convoi, une certaine conception de la sexualité masculine et du comportement des hommes face au sujet de la sexualité et de la santé a été décrite à plusieurs reprises.

Divers documents téléchargés sur le site pendant toute la période de la tournée mettent l'accent sur les problèmes de santé pouvant être à l'origine des difficultés d'érection. L'un d'entre eux, daté du $1^{\text {er }}$ mai 2010, affirmait que « L’impuissance, comme elle est communément désignée, peut être la conséquence d'autres problèmes, comme des cardiopathies (problèmes liés au cœur) et les diabètes. " Le témoignage de l'urologue de la SBU Carlos Antonio da Souza, du 21 mai disait : « Il convient de savoir que la dysfonction érectile peut être un premier symptôme de maladies systémiques plus graves, comme les cardiopathies, l'hypertension artérielle, le cholestérol, des triglycérides élevées et des diabètes, entre autres ». Une note publiée le 29 mai ajoutait que : «Il est très difficile pour un homme de gérer le problème de la dysfonction érectile, qui peut être causée par le stress, des problèmes psychologiques ou peut aussi être le symptôme de maladies comme le diabète et l'hypertension »; et un article du 17 juillet résumait : « La capacité sexuelle a des "ennemis importants", comme l'hypertension, le diabète, la cigarette, le cholestérol élevé, le surpoids et le sédentarisme. " (www.movimentopelasaudemasculina.com.br/ dernier accès le 5 mars 2011).

Le témoignage de l'urologue Renaldo Sacco, publié le 19 août sur le site de ce Mouvement, fait directement référence à l'existence de nouvelles possibilités pour soigner la dysfonction érectile et explique combien elles facilitent une meilleure connaissance de la maladie et de son traitement: " À mesure que nous avons plus de solutions, les hommes consultent plus leurs médecins. Avant l'arrivée des médicaments améliorant l'érection, celle-ci n'était traitée que sur le plan émotionnel, l'on ne prenait en compte que cette dimension. D’autres méthodes sont ensuite apparues comme les injections, puis des médicaments encore plus efficaces sont arrivées. " Outre un plaidoyer sur l'importance des médicaments contre la dysfonction qui ont 
permis de mieux comprendre et diagnostiquer cette maladie, ce texte met en évidence les bienfaits spécifiques du médicament Cialis, produit par le laboratoire Elli Lylli, qui apporte son soutien financier au Mouvement pour la santé masculine: « Le Cialis est le [médicament] le plus efficace en terme de durée, il est efficace jusqu'à 36 heures. " (www.movimentopelasaudemasculina.com.br/ dernier accès le 5 mars de 2011).

La dysfonction érectile est également une raison, selon les témoignages enregistrés sur le site web, incitant les hommes à consulter plus. Le 29 août, l'urologue Antonio Fonseca Neto affirmait que le convoi avait rendu un important service en poussant les hommes à s'informer sur "l'aspect sexuel " qui « est un sujet qui les préoccupe énormément » permettant ainsi de pouvoir aborder les problèmes liés à la santé. (www.movimentopelasaudademasculina.com.br/ accès le 5 mars 2011).

Les différents indices cités montrent que l'accent sur la santé sexuelle en tant que porte d'entrée ou de capture pour le traitement de la santé des hommes est lié à une série de facteurs et de références récurrentes. Un argument constant est la conception de ce que, habituellement, les hommes ne se préoccupent que peu ou prou de leur propre santé, tâche qui est communément décrite comme responsabilité féminine. Néanmoins, ils seraient incessamment préoccupés par leur performance sexuelle, traduite en termes de fonction érectile. En proposant de traiter cette dernière, les urologues ouvriraient la possibilité d'attirer des hommes vers le traitement d'autres maladies. Pour que cette stratégie fonctionne, il serait donc fondamental d'assurer une présence plus représentative et continuelle d'urologues dans les services publics de santé.

Une autre référence fréquente est l'utilisation singulière de la notion de santé sexuelle. On ne saurait ne pas mentionner que ce concept est devenu si important qu'il est officiellement promu par l'Organisation Mondiale de la Santé elle-même. En outre, au-delà du contexte de discussion des droits sexuels, il faut noter qu'il est devenu un élément-clé très souvent associé à des intérêts divers. On observe qu'en plus de vider la notion de santé sexuelle, il la réduit, dans le cas masculin, à la dysfonction érectile, corroborant ainsi l'idée d'une sexualité masculine restreinte à l'érection, presque exclusivement pensée, dans ce contexte, sur des repères hétérosexuels. De cette manière, toute autre forme de perception par rapport à la sexualité masculine est ignorée. 


\section{Une nouvelle onde de médicalisation}

À l'aune du panorama décrit jusqu'à présent, l'on peut argumenter qu'une nouvelle onde de médicalisation de la sexualité masculine commence à être observée principalement dans la promotion de diagnostics de dysfonction sexuelle et d'andropause ou Déficit androgénique lié à l'âge (DALA) (Rohden 2011). Barbara Marshall et Stephen Katz (2002) soulignent que, au XXe siècle, le processus de médicalisation de la sexualité se concentre sur l'homme et circonscrit la sexualité masculine à la dysfonction érectile. Par le biais d'une problématisation plus générale articulant sexualité et âge comme des dimensions fondamentales du sujet moderne, il met l'accent sur l'importance des cultures de style de vie de la fin du XXe siècle en insistant sur la santé, l'activité et le non au vieillissement en une procédure qui va être à l'origine d'un vaste champ d'études et d'intervention sur la capacité de pénétration de l'organe sexuel masculin. La grande nouveauté, selon ces auteurs, est que l'on passe d'une conception admettant le déclin de la vie sexuelle au cours du temps et où l'activité sexuelle dans la vieillesse est péjorativement suspecte à une autre où il est obligatoire d'avoir une bonne performance sexuelle jusqu'à la fin de sa vie. Plus encore, où l'on affirme que l'activité sexuelle est bien une condition nécessaire pour une vie en pleine santé et que la capacité érectile définirait la virilité pendant tout le cours de la vie masculine. C'est précisément dans ce contexte qu'a surgi le Viagra (citrate de sildénafil), médicament du laboratoire Pfizer destiné à faciliter et maintenir l'érection, qui illustre le développement d'une science moléculaire de la sexualité (Marsall et Katz 2002; Marshall 2006).

Pour garantir le succès de ce nouveau médicament, il a fallu transformer la dysfonction érectile en problème pouvant toucher n’importe quel homme, dans n'importe quelle phase de sa vie et, en même temps, garantir qu'un remède capable de résoudre ou de prévenir cette difficulté soit disponible. En ce sens, le Viagra intégrerait la gamme bien plus vaste de ce que l'on appelle les médicaments de style de vie ou de confort, destinés à améliorer la performance individuelle, un marché en franche expansion. En outre, Pfizer a également travaillé à promouvoir l'idée de la dysfonction érectile comme topique acceptable dans le discours public, ce qui conduirait à une plus grande demande de traitement (Lexchin 2006).

Cela impliquait la propagation d'une idée de masculinité en crise, illustrée surtout par la métaphore de l'érection. La notion de ce que cette dernière, 
symbole de la virilité et de l'identité masculine, est effectivement instable, sujette à différents types de désagréments, semble acquérir de plus en plus de notoriété. Et, c'est justement pour combattre ce manque de contrôle ou cette imprévisibilité du corps masculin que l'industrie offre un recours tel que le Viagra, capable de garantir l'expectative d'une performance toujours meilleure (Grace et al 2006; Vares et Braun 2006; Loe 2001).

Un facteur important dans ce processus a été la présence croissante des urologues et leur rôle fondamental dans l'institutionnalisation du champ de la médecine sexuelle, moyennant la création d'organisations, de conférences, de centres de formation, de journaux scientifiques, de cliniques et de départements médicaux (Giami 2004 ; Tiefer 2006). Le « succès » de ces professionnels dans la promotion de cette nouvelle possibilité de médicaliser la sexualité masculine s'étendrait aussi au diagnostic de l'andropause ou DALA. Défini comme « maladie » qui affecterait les hommes à partir de 35-40 ans, dernier est caractérisé par la perte de la libido ou du désir sexuel, la diminution de la masse musculaire, la perte d'énergie, la dépression, la dysfonction érectile, entre autres symptômes dont la cause est le déclin de la production de testostérone. Même s'il s'agit d'une maladie reconnue comme organique et traitable depuis les années 1930, ce n'est qu'à partir des années 60 que son traitement a commencé à mettre en avant les problèmes d'ordre sexuel (Marshall 2007). Dès les années 9o, l’utilisation de testostérone gagne du terrain et l'on donne de plus en plus d'importance à la restauration de la performance sexuelle, en parallèle aux traitements pour la dysfonction érectile.

Au Brésil, les urologues, représentés par la SBU, ont contribué de manière singulière à ce qu'une attention spéciale soit donnée à l'andropause et à la dysfonction érectile. Cette société s'est consacrée à la réalisation d'une série de campagnes et autres événements sur le modèle du Mouvement pour la santé masculine, réalisé en 2010, visant à sensibiliser la population au besoin de prévention et de traitement de troubles comme la dysfonction érectile, l'andropause et les maladies de la prostate. L'Unité Mobile du Mouvement (un semi-remorque équipé avec des cabinets et une équipe composée de trois urologues et d'autres professionnels de santé), sponsorisé par le laboratoire Eli Lilly, a parcouru les grandes capitales du pays pour offrir une orientation médicale gratuite. 
La promotion de ce type de campagnes et le fait d'attirer l'attention publique sur le DALA et la dysfonction érectile illustrent bien que, ces deux dernières décennies, un nouveau regard sur la masculinité s'est façonné via la pharmacologisation de la sexualité. Cela passe soit par le biais de drogues facilitant l'érection, soit par la prescription de testostérone. Bien qu'une certaine perspective critique apparaisse, surtout avec la divulgation de données remettant en cause l'efficacité et la sécurité de ces thérapies, l'on peut supposer que la médicalisation a prévalu, aussi bien dans la pratique médicale que dans les représentations de sens commun qui se consolident. Dans le cas spécifique de la conjonction entre vieillissement et sexualité, il convient de noter que la promotion des nouvelles drogues et ressources chemine côte à côte avec la promotion de modèles de comportement centrés sur la valorisation du corps jeune, sain et sexuellement actif.

\section{Le champ des savoirs sur la sexualité et le processus de médicalisation}

Les données présentées jusqu'à présent suggèrent une différence importante entre l'approche de la sexualité masculine dans le contexte des campagnes contre la propagation de la syphilis et celui, récent, des politiques sur la médecine sexuelle. Plutôt que de décrire ici un parcours historique continu, nous entendons révéler des points de rupture, du point de vue analytique. Ainsi, il nous faut avoir recours à certains des auteurs les plus importants dans ce domaine pour souligner les transformations mises en relief dans cet article.

De nombreux chercheurs ont montré comment la sexualité, en particulier dans le contexte de la modernité, peut fournir des informations importantes sur les individus. Plus particulièrement à partir du XIX ${ }^{\mathrm{e}}$ siècle, il est possible de voir surgir un intérêt croissant pour le sexe qui se traduit par le développement d'un ensemble de nouveaux savoirs comme la gynécologie, la psychanalyse et la sexologie. Tous sont intimement liés à la production de nouvelles subjectivités en même temps qu'ils reflètent les changements observés.

M. Foucault (1988) associe ce processus à une nouvelle approche et au traitement disciplinaire du corps. Plus encore, il identifie dans l'histoire de l'Occident un passage fondamental associé à l'invention d'une sexualité de culpabilité, à un moment unique, de « répression " et de "libération ". L’individu est perçu comme capable de déceler sa vérité à partir de ce qui est 
dit ou non dit, réprimandé en relation au sexe. Cette approche nous pousse à considérer comment la sexualité a constitué un nœud de représentations fondamentales autour duquel se sont articulés des conflits se référant aux contextes sociaux, politiques et économiques depuis le milieu du XIX ${ }^{e}$ siècle, quand la médecine acquiert une place importante.

Dans cette perspective, J. Weeks (1985) analyse l'émergence de la sexologie qui, à l'origine, est immergée dans la production des gynécologues et autres spécialistes. En traitant particulièrement de la sexologie, de sa constitution en tant que science et de son usage pour définir de nouvelles identités culturelles, cet auteur argumente aussi dans le sens d'une perméabilité des discours et, au-delà, des effets inespérés que les propositions scientifiques peuvent avoir. Weeks part du principe que la sexualité est une invention sociale localisée, un produit de mouvements historiques exprimant les forces sociales en jeu dans chaque contexte.

La sexologie émerge comme un domaine de connaissance spécifique dédié au contrôle des individus en s'efforçant de définir ce qui peut-être considéré comme normal, en terme de relation avec le sexe opposé. Pour Weeks (1985), la sexualité est un champ de bataille, de construction de discours sociaux, et l'invention de la sexologie finit par avoir des conséquences diverses. Elle est également à la source de discours libéraux et permet de créer des identités sociales fondées sur les « découvertes » des médecins, comme dans le cas des mouvements féministe et homosexuel. Toutefois, il convient de souligner le rôle de la sexologie dans la réaffirmation des différences entre homme et femme, de l'attraction naturelle pour le sexe opposé et dans la création même du concept d'homosexualité - toujours lié à un signe biologique. Ces « découvertes scientifiques " apparaissent aussi à Weeks (1985) comme le résultat de demandes sociales, d'un panorama de changements exigeant de nouveaux codes pour gérer les rapports entre hommes et femmes et définir ce qui est acceptable dans la société qui se constitue. Pour cet auteur, les sexologues réussissent à traduire en termes théoriques ce qui est ressenti en tant que problèmes sociaux concrets, comme l'existence même de la sexualité des enfants et des adolescents ou la relation entre maternité et sexualité féminine. En réalité, une préoccupation plus importante envers les changements observés dans le rapport homme-femme serait au centre des spéculations produites sur la bisexualité, le travestissement, l’intersexualité et l'instinct de reproduction. 
Weeks (1985) souligne que ce constat révèle une difficulté à définir les limites entre sexologues et réformateurs sexuels, eugénistes et moralistes. En même temps qu'ils apportent des réponses à l'exigence de nouvelles limites dans les relations entre hommes et femmes et de ce qui est normal en termes de sexualité, ils finissent par fournir de nouvelles bases scientifiques aux conflits sociaux. Les différences ont maintenant des bases biologiques, les pratiques déviantes sont classifiées, les féministes utilisent les arguments scientifiques, les homosexuels acquièrent une identité qui ne leur avait jamais été reconnue dans un autre contexte.

La complexité du champ des savoirs et les interventions autour du sexe ont aussi été la cible du travail classique de A. Béjin (1987a ; 1987b). L’une de ses contributions est l'hypothèse de ce qu'il existerait deux sexologies. La première aurait été produite dans la seconde moitié du XIX ${ }^{e}$ siècle, période où paraissent des œuvres de référence comme Psychopatia sexualis, édité par Heirich Kann en 1844 et un volume publié sous ce même titre par Kraft-Ebing en 1886. Cette " protosexologie " avait pour objet la nosographie, en contraste avec la thérapie, et privilégiait les maladies vénériennes, la psychopathie de la sexualité et l'eugénisme. La seconde aurait émergé à partir des années 20 et un point de référence important en serait l'œuvre de W. Reich, qui a débuté ses publications sur la fonction de l'orgasme. L'édition de la première étude de A. Kinsey en 1948 aiderait à concrétiser l'orgasme comme thème central de cette nouvelle sexologie (Béjin, 1987a).

Cet auteur souligne également que la protosexologie se concentrait sur les difficultés relatives au fonctionnement de la sexualité reproductive, comme les maladies vénériennes, les « aberrations sexuelles » et les techniques contraceptives et n'était pas très encline à se différencier des autres branches de la médecine, comme la psychiatrie, la médecine légale ou l'urologie. En revanche, la sexologie actuelle n'aurait cesse de chercher son autonomie face aux autres disciplines, en particulier sur la base de l'objet particulier que serait l'orgasme et d'une norme fondamentale, « l'orgasme idéal ». En outre, la différence de base entre ces deux phases se doit au fait que :

« La protosexologie entendait étudier (et, bien souvent combattre) les anomalies, bien qu'elle puisse à peine dévoiler la norme qu'elle mettait en avant (essentiellement, le coït hétérosexuel reproductif). « L'orgasmologie » adopte un processus totalement différent ; elle commence par élaborer sa norme pour 
en déduire des anomalies qu'elle se déclare immédiatement capable de soigner. Comme la norme - à l'exemple de « l'orgasme idéal » de la «Constitution » de Master Jonhson - représente souvent un objet empiriquement inaccessible, ces anomalies ne manquent pas. On peut observer que les sexologues modernes ne se réfèrent pas à ces anomalies, ces " aberrations".

En réalité, ils substituent cette opposition marquée entre normalité et anomalie par un ensemble de dysfonctions. Face à la norme de l'orgasme céleste, nous souffrons tous de " dysfonctionnements sexuels ». (Béjin, 1987a :228)

Cet auteur (1987a) souligne encore que l'une des conséquences de ce fait est la génération d'une importante " clientèle ", de plus en plus nombreuse, pour les sexologues contemporains, à l'inverse des pionniers qui ne traitaient que les " pervertis » et les porteurs de maladies vénériennes. Ce mouvement a favorisé la création de cliniques et d'un enseignement spécialisés. De plus, alors que la protosexologie avait sommairement développé son éthologie, ne permettant qu'un contrôle a posteriori et répressif, en lien avec les prisons et les asiles, la sexologie actuelle a raffiné son éthologie et développe des moyens de contrôle a priori et a posteriori, qui se traduisent par les thérapies de l'orgasme et la prophylaxie des dysfonctionnements sexuels. Une fonction pédagogique toujours plus centralisée sur l'information est mise en avant.

Dans son livre Disorders of desire, Janice Irvine (2005) montre comment la sexologie s'est constituée aux États-Unis entre les années 40 et 80, en soulignant les aspects multidisciplinaires, la persécution et la controverse. À partir du travail d'A. Kinsey, cette auteure révèle les impasses des processus de professionnalisation, de légitimation culturelle et de création d'un marché autour du sexe. Les tensions politiques et les variations des contextes historico-culturels ont eu une influence importante sur la recherche, les interventions et l'acceptation de nouvelles références sur la sexualité. En outre, les débats ont été orientés autour de la distinction entre une " sexologie scientifique ", s'appuyant sur les paramètres méthodologiques de la science, la pratique et l'autorité de la médecine et une " sexologie humaniste ", fondée sur la reconnaissance de la sexualité comme objet de réalisation personnelle, de connaissance de soi et de satisfaction individuelle.

Dans le cas de la France, Giami et Columby (2001), mentionnent comme référence la création en 1931, de l'Association d'Études sexologiques sous l'influence d'Édouard Toulouse. À l'époque, la sexologie s'inscrivait dans un 
courant néo-malthusien hygiéniste. Jusqu'aux années 6o, dans ce pays, la sexologie était caractérisée par un ensemble d'initiatives diverses issues de différentes perspectives. Elle recouvrait les volets procréatifs et érotiques de la sexualité et s'intéressait aux aspects individuels et collectifs. Mais ce cadre à beaucoup évolué avec la vente de pilules anti-contraceptives sur le marché, la libération de l'avortement et le développement de l'éducation sexuelle de masse.

Actualisant une histoire récente de la sexologie, Giami (2000) souligne deux points fondamentaux. Le premier se réfère à la discussion sur la médicalisation de la sexualité et le second touche au débat plus actuel autour de la « dysfonction érectile ». Sa perspective accompagne d'autres travaux analysant ce champ, où il est coutume de montrer la différence entre la sexologie du XIX ${ }^{e}$ siècle et celle du XXe siècle. Alors que la première mettrait en évidence l'aspect pathologique de la sexualité, celle du XXe siècle (d'après les travaux d'A. Kinsey) se concentrerait de plus en plus sur la question de la "santé sexuelle ».

Pour ce qui est du processus contemporain de médicalisation de la sexualité, Giami $(2000,2001,2004)$ suggère une suprématie des approches ou explications des problèmes sexuels en relation à la psychologie. Il identifie la médicalisation de l'impuissance masculine au cours d'un processus débutant dans les années 80 avec les découvertes scientifiques, comme l'effet de la papavérine sur l'érection. À partir d'alors, un groupe d'urologues a continué à proposer des définitions plus restrictives que celles du champ de la médecine organiciste, abandonnant les conceptions psychologiques qui avaient dominé au long des décennies précédentes et prenant ses distances de l'approche chirurgicale (auparavant dominante en urologie). Un reflet de ce changement est la création, en 1993, par le National Institute of Health (NIH), aux États-Unis, du terme « dysfonction érectile » qui vient remplacer " impuissance sexuelle ». Ces mêmes urologues se sont ensuite intéressés au terrain de l'épidémiologie et ont établi de nouvelles données sur la prévalence de l'impuissance.

En dépit de la réserve sur le maintien du terme « impuissance » par la littérature médicale, on peut observer que ce dernier fait maintenant référence à une conception plus large de la sexualité, incluant les dimensions psychologiques et relationnelles. En ce qui concerne le terme " dysfonction érectile » il semble plus restreint aux dimensions biologiques et physiologiques de 
fonctionnement du pénis, considéré comme un facteur externe à l'homme et hors de son contrôle. Giami (2004) mentionne cependant une tendance de la substitution de l'éthologie psychologique de l'impuissance par une éthologie organique représentée par la dysfonction érectile.

La dysfonction érectile est définie comme « l'incapacité pour l'homme d'obtenir un pénis en érection comme partie du processus multiforme de la fonction sexuelle. " (HIH conference 1993, p.83, in : Giami, 2004. Ainsi segmentée, la dysfonction érectile permettrait d'établir des niveaux de sévérité en incluant, sous une surveillance de la maladie, un large spectre de fonctionnement sexuel. Selon Giami (2004:102) : « La dysfonction érectile se différencie de l'impuissance par une diminution de la maîtrise de l'érection, à éthologie organique, et par une extension de la prévalence incluant des formes plus légères " (Traduction de l'auteur).

Face à ce nouvel objet clinique qui venait de naître, l'industrie pharmaceutique a rapidement apporté une réponse sous forme de médicament, le Viagra (1998), contribuant à la diffusion des conceptions sur cet objet et ses transformations. Giami (2004) souligne la nécessité de comprendre le développement du Viagra comme un phénomène culturel sur différents plans. En ce sens, il met en relation le discours sur la stimulation sexuelle, sous l'influence du Viagra, et ceux de prévention relatifs au SIDA, pour souligner un paradoxe : alors que le discours sur le SIDA se focalise sur l'activité sexuelle des " jeunes ", la multiplication des partenaires, les homosexuels et les pratiques à risque (comme la pénétration anale), le discours sur la dysfonction érectile s'applique à la pénétration génitale du couple hétérosexuel et s'adresse aux hommes âgés de plus de 40 ans. Deux approches de la sexualité qui s'ignorent mais qui en fait se montrent complémentaires : alors que l'impuissance touche la sexualité conjugale, le risque lié aux maladies sexuellement transmissibles menace la vie sexuelle hors du mariage (Giami 2004 :103).

Nous avons donc une relecture par les médias de la signification du Viagra mettant en relief son rôle d'aphrodisiaque et sa fonction récréative dans l'activité sexuelle. Un troisième sens peut-être proposé, si nous le considérons comme un médicament améliorant la qualité de vie. Giami (2004) conclut en soulignant l'apparition d'un processus paradoxal de « démédicalisation " graduée de la sexualité dans la mesure où les normes de santé et de sexualité passeraient toujours plus par l'exclusion de la prescription médicale 
au profit du contrôle de l'industrie pharmaceutique. Une des raisons invoquées reposerait sur la possibilité de financement de la recherche et de nouvelles opportunités professionnelles. La soi-disant " médecine sexuelle » illustrerait un processus de consolidation de ce lien, représenté principalement par les urologues.

Il est possible de noter que même si le champ d'intervention de la sexualité se caractérise par la coexistence de différentes professions, l'approche biomédicale s'est imposée comme prédominante principalement à partir de la fin des années go et de la prescription de ce que l'on appelle des médicaments pro-sexuels. Ce cadre est associé à une nouvelle configuration d'idées et à un nouvel ensemble d'interventions concrètes que nous pourrions qualifier de nouvelle norme basée sur la performance sexuelle satisfaisante.

L'objectif des nouveaux discours et pratiques sur le sexe semble être de maximiser l'exercice de la fonction corporelle, en laissant croire que l'aspect relationnel/social de la sexualité serait éloigné des intérêts des nouveaux scientifiques du sexe. L’idée que les problèmes liés à la sexualité découlent en grande partie "d'absence » ou " d'insuffisance » de l'exercice sexuel est devenue prépondérante. $\mathrm{Si}$, pour les sexologues du XIX ${ }^{\mathrm{e}}$ siècle, la question pertinente était " l'excès sexuel " des femmes " nymphomanes " ou des hommes " pervertis ", le problème est maintenant lié au " manque ". L'individu moderne semble condamné à faire la preuve de sa capacité sexuelle pour pouvoir prouver son identité de sujet épanoui, satisfait et heureux. Les discours sur l'usage des médicaments, en particulier, se sont largement inspirés de cette nouvelle approche.

Il devient de plus en plus clair que l'idée de " santé sexuelle " gagne du terrain. Bien qu'elle fasse parfois référence aux droits et à la notion de bienêtre individuel, ce qui est crucial dans cette notion, c'est l'établissement d'un nouveau paramètre de performance que l'individu d'aujourd'hui doit atteindre. Ce dernier paraît être obligé à jouir d'une activité sexuelle régulière, intense et très performante. Cependant, pour atteindre ce nouveau seuil, il faut avoir recours à des agents externes qui feront le diagnostic de la performance et prescriront les solutions adaptées. La responsabilité est donc transférée à ces agents externes, comme les urologues, qui s'occuperont du bon développement des fonctions sexuelles. Surgit alors l'image d'un individu soumis aux fonctions corporelles et à leur bon fonctionnement, sur la base de standards qui sont définis en dehors de lui. De plus, l'on construit un 
lien fort entre santé sexuelle et santé en général, ce qui, dans le cas masculin, est représenté par la capacité d'érection qui devrait toujours être maximisée.

Ces arguments aident à analyser les différences entre les deux investissements dans la santé et la sexualité masculines évoquées dans ce travail. Comme nous pouvons le voir, la production de savoirs sur la sexualité qui se développe de la fin du XIX ${ }^{e}$ siècle jusqu'au début du XXIe siècle est marquée par un intérêt croissant pour la notion de santé et d'amélioration de la performance individuelle, caractéristiques principales pour mieux comprendre les initiatives entreprises par les urologues liés au domaine de la médecine sexuelle, que l'on observe aussi au Brésil.

\section{Perspectives concernant la médicalisation de la sexualité masculine}

La récente médicalisation de la sexualité masculine, centrée sur l'ascension des diagnostics de dysfonction érectile et DALA, montre une série de contrastes intéressants par rapport au type de médicalisation qui a caractérisé le processus concernant la syphilis dans les premières décennies du $\mathrm{XX}^{\mathrm{e}}$ siècle. Il convient d'observer que ces deux situations présentent sans aucun doute des contextes sociaux extrêmement distincts qui se traduisent par exemple en espérances de vie très différentes et en politiques publiques ayant des intentions bien contrastées. D’une manière générale, si dans un premier temps la question de la santé entre dans un registre plus collectiviste, de préoccupation envers la nation, dans le second, la référence forte se confond avec une idéologie plus individualiste, allant très souvent dans la direction contraire des discussions sur la promotion de la santé via la perspective des droits et de la citoyenneté.

Il serait possible d'indiquer certaines " permanences » ou points communs, comme l'idée courante de ce que les hommes sont énormément préoccupés par le sexe, ce qui exigerait des formes d'administration distinctes. Pourtant, même sur ce point nous aurions des différences significatives. Si, dans les campagnes contre la syphilis, la question se traduisait en termes d'autocontrôle des " excès " sexuels au profit du bien collectif ou de la nation, actuellement le point central semble être la promotion du soin de soi, comme manière d'atteindre un accomplissement personnel projeté. En outre, la syphilis apparaissait sous le registre d'une maladie externe et fragilisante qui pourrait affecter les hommes, alors que la médicalisation via la 
reconnaissance de problèmes fonctionnels et biochimiques renvoie à des difficultés inhérentes au corps de l'individu lui-même et à son fonctionnement. Alors que le discours sur la syphilis accentuait les dangers de la dégénération collective, celui qui aborde la santé sexuelle dans le contexte contemporain met surtout l'accent sur une régénération de la puissance sexuelle individuelle.

La prédominance de ce courant plus individualiste, calqué sur l'amélioration de la performance et de la fonctionnalité et les résolutions pharmacologiques nous renvoie aux discussions récentes sur les processus de bio-médicalisation, associés à la conformation d'une nouvelle culture, ou " régime de vérité ", centrée sur la responsabilisation individuelle. Dans ce contexte, la préoccupation pour la santé devient un attribut moral de l’individu, lequel doit être informé des nouvelles connaissances, des pratiques de soins de soi, de prévention et de traitement des maladies et disposé à consommer les ressources actuellement disponibles (Clarke et al 2009).

Cette argumentation prend de l'importance dans l'œuvre de Nikolas Rose (2007) qui s'interroge sur le rôle des sciences de la vie dans la production de vérités et de subjectivités contemporaines. Sa discussion des concepts de molécularisation, d'optimisation, de subjectivation, d'expertise et de bioéconomie est importante pour comprendre la dynamique des transformations impliquant la notion de corps sain centrée sur l'autogestion personnelle. Quand il parle de molécularisation, Rose met l'accent sur le passage d'une conception biomédicale centrée sur le corps à celle qui, maintenant, se spécialise au niveau moléculaire, ce qui pourrait être décrit en termes d'une nouvelle biopolitique. L’optimisation est présentée comme l'utilisation des technologies médicales contemporaines non seulement pour guérir des pathologies mais encore pour contrôler les processus vitaux du corps et de l'esprit. Ces technologies de l'optimisation sont associées à l’idée de l'accomplissement comme quelque chose dirigé vers le futur et à la possibilité de création d'individus consommateurs de ces nouveaux désirs et de ces nouvelles possibilités de contrôle de la vie. Le concept de subjectivation sert à décrire le processus par lequel le sujet est amené à croire que la promotion de la santé est une question personnelle, d'autogestion et de responsabilité.

Cela serait associé à la conversion de la santé en tant que valeur éthique importante dans la société occidentale, à partir de la seconde moitié du $\mathrm{XX}^{\mathrm{e}}$ siècle et, de façon plus contemporaine, à la formation d'une nouvelle 
éthique. Il s'agit d'une " éthopolitique », soit de la tentative de modeler la conduite des êtres humains par le biais d'une action sur les sentiments, les croyances et les valeurs axée sur la manière dont ils devraient juger et agir sur eux-mêmes et leur corps, en visant le futur. L'expertise, quant à elle, devient importante par rapport aux pratiques de biopouvoir émergentes qui sont liées à de nouvelles formes d'autorité. Les actuels " experts de la propre vie " ne se distinguent plus en fonction de la guérison de maladies, mais de leur capacité à améliorer les arts de l'autogouvernement. Médecins et autres professionnels de santé formeraient ce champ d' " experts somatiques ", capables d'orienter les individus vers la recherche de l'amélioration ou de l'optimisation de leurs potentialités. (Rose 2007).

Il est possible de suggérer, dans le cas de la médicalisation de la sexualité masculine, que ces phénomènes plus généraux peuvent être articulés autour de certaines transformations importantes. En ce qui concerne le développement de nouvelles spécialités et de nouveaux " experts ", l'on pourrait citer le contraste entre l'insuccès de l'institutionnalisation de l'andrologie, telle que proposée par José de Albuquerque et l'ascension de l'urologie, lorsqu'elle se convertit, du moins en partie, en médecine sexuelle. Bien qu'actuellement la Société brésilienne d'Urologie compte sur un département d'Andrologie, le plus important est que l'urologie elle-même s'est mise en évidence comme spécialité légitime dans le soin de la sexualité masculine. Si la proposition d'une science du sexe masculin, calquée sur des paramètres moraux et scientifiques n'a pas avancé au cours des années 1930, la nouvelle pharmacologie sexuelle se montre de plus en plus présente.

Il convient encore de noter que ce nouveau processus de médicalisation de la sexualité masculine ne se produit que lorsque la santé, notamment la santé sexuelle, commence à se convertir en bien valorisé en tant que tel, en contraste avec la médicalisation qui mettait en avant la maladie. Ce n'est que dans ce nouveau contexte que le discours public des urologues paraît connaître plus de succès. Cela revient à dire que, pour les urologues brésiliens, les hommes ne seraient attirés dans les services de santé pour traiter de maladies que lorsque des possibilités d'amélioration de leur potentialités et plus particulièrement de la fonction sexuelle sont impliquées.

Pour conclure, si dans le processus de médicalisation et contrôle par le biais de la syphilis, l'accès aux hommes passait par une maladie, externe, contagieuse et affaiblissante, qui porterait préjudice à la nation elle-même, 
de nos jours la médicalisation opère par le biais de la menace de la faible performance et du besoin d'améliorer la fonctionnalité liée à des facteurs moléculaires. Il ne s'agirait plus de l'autocontrôle nécessaire aux citoyens des États émergents mais du soin de soi incontournable pour les individus responsabilisés pour leur santé, leur bien-être et leur accomplissement. On pourrait parler d'une médicalisation " par l'intérieur » et " pour l'amélioration », liée à la promotion de la santé en tant que valeur culturelle et bien de consommation.

Reçu le 4 juillet de 2014, approuvé le 1 Décembre, 2014

\section{Références}

ASSOCIAÇÃO Brasileira para os Estudos das Inadequações Sexuais.

Disponible sur le site Web www.abeis.org.br, 23/03/2011.

BAYER Schering Pharma. 2008. A saúde sexual como portal da saúde do homem. BÉJIN, Andre. 1987a. "Crespúsculo dos psicanalistas, manhã dos sexólogos”. In: Ph. Ariès, et A. Béjin (orgs.), Sexualidades ocidentais. São Paulo: Brasilense. pp.211-235 . 1987b. "O poder dos sexólogos e a democracia sexual". In: Ph. Ariès \& A. Béjin (orgs.), Sexualidades ocidentais. São Paulo: Brasilense. pp.236-254. BUCHALLA, Paula A. 2009. "A Revolução Azul: dez anos depois do lançamento do Viagra, a impotência deixou de ser um fantasma masculino". Veja, 19 março 2009. . 2008. "Sexo, Remédios e...felicidade. Para o médico americano, não há nada de errado em um homem recorrer à química para melhorar o desempenho na cama. É bom até para elas. Basta ter critério". Veja, 9 janeiro 2008.

CÂMARA dos Deputados. Disponible sur le site Web www2.camara.gov.br| internet/homeagencia/matérias.html, 11/03/2011.

CARRARA, Sérgio. 1996. Tributo a Vênus - a luta contra a síflis no Brasil, da passagem do século aos anos 40. Rio de Janeiro: Ed. Fiocruz. ; RUSSO, Jane; FARO Livi. 2009. "A política de atenção à saúde do homem no Brasil: os paradoxos da medicalização do corpo masculino". Physis, 19(3): 659-678. 
CLARKE, Adele E.; SHIM, Janet; MAMO, Laura; FOSKET, Jennifer: FISHMAN, Jennifer. 2009. Biomedicalization: technoscience and transformations of health and illness in the U.S. Durham: Duke University Press.

COUTO, Marcia T.; PINHEIRO, Thiago; VALENÇA Otávio. 2010. “O homem na atenção primária à saúde: discutindo (in)visibilidade a partir da perspectiva de gênero". Interface. Comunicação, Saúde e Educação,14: 257270.

FARO, Livi Testoni Ferreira; CHAZAN, Lilian Krakowiski; ROHDEN, Fabíola; RUSSO, Jane. 2013. "Homem com "H”. Ideais de masculinidade (re) construídos no marketing farmacêutico". Cadernos Pagu, 40: 287-321.

FOUCAULT, Michel. 1988. História da sexualidade I: a vontade de saber. Rio de Janeiro: Graal.

GIAMI, Alaim. 2004. «De l'impuissance à la dysfonction éretile: destins de la médicalization de la sexualité”. In: D. Fassin et D. Memmi (org.), Le gouvernemant des corps. Paris: Éditions EHESS. pp.77-108.

. 2002. "Sexual health: the emergence, develepment, and diversity of a concept”. Annual Review of Sex Research, XIII: 1-35.

. 2001. "Changing relations between medicine, psychology and sexuality: the case of male impuissance". The Journal of Social Medicine,37: 263-272.

; COLOMBY, Patrick. 2001. “Profession sexologue?” Sociétés

Contemporaines, 41/42: 41-63.

GOMES, Romeu (org.). Saúde do homem em debate. Rio de Janeiro: Fiocruz. . 2008. Sexualidade masculina, gênero e saúde. Rio de Janeiro: Fiocruz. .; SCHRAIBER, Lilian; COUTO, Marcia T. 2011. "O atendimento à saúde de homens: estudo qualitativo em quatro estados brasileiros”. Physis,21: 113-127.

GRACE, Victoria, POTTS, Annie, GAVEY, Nicola; VARES, Tiina. 2006. “The discursive condition of Viagra”. Sexualities, 9(3): 295-314.

IRVINE, Janice. 2005. Disorders of desire: sexuality and gender in modern American sexology. Philadelphia: Temple Univ. Press.

Isto É ."Porque ele não vai ao médico?” 9 julho 2009.

LEXCHIN, Joe. 2006. "Bigger and better: how Pfizer redefined erectile dysfunction". Plosmedicine, 3(4): 1-4.

LOE, Meika. 2001. "Fixing broken masculinity: Viagra as a technology for the production of gender and sexuality". Sexuality and Culture, 5(3): 97-125. 
MARSHALL, Barbara. 2007. "Climateric redux?: (Re)medicalizing the male menopause". Men and masculinity, 9: 509-529. . 2006. "The new virility: Viagra, male aging and sexual function". Sexualities, 9(3): 345-362. ; KATZ, Stephen. 2002. "Forever functional: sexual fitness and the ageing male body". Body and Society, 8(43): 43-70.

MEDRADO, Benedito; LYRA, Jorge; AZEVEDO, Mariana; GRANJA, Edna; VIEIRAS, Sirley. 2009. Princípios, diretrizes e recomendações para uma atenção integral aos homens na saúde. Recife: Instituto Papai.

MEDRADO, Benedito; LYRA, Jorge; AZEVEDO, Mariana. 2011. "Eu não sou só próstata, eu sou um homem?' Por uma política pública de saúde transformadora da ordem de gênero". In: Romeu Gomes (org.), Saúde do homem em debate. Rio de Janeiro: Fiocruz. pp. 39-74.

MOVIMENTO pela Saúde Masculina. Disponible sur le site www. movimentopelasaudemasculina.com.br, 5/03/2011.

O Globo ."Coisa de homem: por vergonha ou falta de recursos, maioria não procura ajuda contra doenças masculinas." 17 agosto 2008.

PINHEIRO Thiago, COUTO, Marcia; SILVA, Geórgia. 2011. "Questões de sexualidade masculina na atenção primária à saúde: gênero e medicalização". Interface, 15: 845-858.

ROHDEN, Fabíola. 2012 "Capturados pelo sexo: a medicalização da sexualdidade masculina em dois momentos". Ciência e Saúde Coletiva, 17: 2645-2654.

. 2011. "O homem é mesmo a sua testosterona: promoção da andropausa e representações sobre sexualidade e envelhecimento no cenário brasileiro". Horizontes Antropológicos, 17: 161-196. . 2009. Uma ciência da diferença: sexo e gênero na medicina da mulher. $2^{2}$. Edição. Rio de Janeiro: FIOCRUZ. . 2009. "Diferenças de gênero e medicalização da sexualidade na criação do diagnóstico das disfunções sexuais". Estudos Feministas, 17(1): 89-109. ROSE, Nicolas. 2007. The politics of life itself: biomedicine, power, subjectivity in the twenty-first century. Princeton: Princeton University Press. ROSENFELD, Dana; FAIRCLOTH, Christopher A. 2006. Medicalized masculinities. Philadelphia: Temple University Press. 
RUSSO, Jane; ROHDEN, Fabíola; TORRES, Igor; FARO, Livi T F; NUCCI, Marina; GIAMI, Alaim. 2011. Sexualidade, ciência e profissão no Brasil. Rio de Janeiro: CEPESC.

SCHRAIBER Lilian; GOMES, Romeu; COUTO, Marcia. 2005. "Homens e saúde na pauta da Saúde Coletiva”. Ciência e Saúde Coletiva,1o(1): 7-17.

SCHRAIBER, Lilian; FIGUEIREDO, Wagner. 2011. "Integralidade em saúde e os homens na perspectiva relacional de gênero". In: R. Gomes (org.), Saúde do homem em debate. Rio de Janeiro: Fiocruz. pp.19-38.

SOCIEDADE Brasileira de Urologia. Disponible sur le site http://www.sbu.org. br, 17/02/2009, 15/09/2009, 20/09/2009, 21/06/2010.

TIEFER, Leonore. 2006. “The Viagra phenomenon”. Sexualities, 9(3): 273-294. . 2004. Sex is not a natural act. Cambridge: Westview Press.

VARES, Tiina; BRAUN, Victoria. 2006. "Spreading the word, but what word is that? Viagra and male sexuality in popular culture". Sexualities, 9(3): 315-332.

WEEKS, Jeffrey. 1985. Sexuality and its discontents. London/New York: Routledge/Keagan Paul.

\section{Fabíola Rohden}

Département d'Anthropologie, l'Université Fédérale du Rio Grande do Sul (UFRGS)

fabiola.rohden@gmail.com 